\title{
Versatility of field theory motivated nuclear effective Lagrangian approach
}

\author{
P. Arumugam, B. K. Sharma, P. K. Sahu, and S. K. Patra \\ Institute of Physics, Sachivalaya Marg, Bhubaneswar - 751 005, India. \\ Tapas Sil, M. Centelles, and X. Viñas \\ Departament d'Estructura i Constituents de la Matèria, Facultat de Física, \\ Universitat de Barcelona, Diagonal 647, 08028 Barcelona, Spain
}

\begin{abstract}
We analyze the results for infinite nuclear and neutron matter using the standard relativistic mean field model and its recent effective field theory motivated generalization. For the first time, we show quantitatively that the inclusion in the effective theory of vector meson self-interactions and scalar-vector cross-interactions explains naturally the recent experimental observations of the softness of the nuclear equation of state, without losing the advantages of the standard relativistic model for finite nuclei.
\end{abstract}

PACS numbers: 21.30.Fe, 21.65.+f, 26.60.+c, 21.60.-n 


\section{INTRODUCTION}

In the quest for a unified model describing both finite nuclei and nuclear matter properties, the relativistic mean field (RMF) approach to quantum hadrodynamics 1] has become a very popular tool. The original linear $\sigma-\omega$ model of Walecka [1] was complemented on an empirical basis with cubic and quartic non-linearities [2] of the $\sigma$ meson to have proper results. We shall refer to this approach as standard RMF model. In recent years novel approaches based on modern concepts of effective field theory (EFT) and density functional theory (DFT) for hadrons, in different implementations, have been developed for the relativistic nuclear many-body problem [3, 4, 5, 6, 7, 8]. One of the goals is to overcome the ad hoc nature of the previous models and the presence of uncontrolled approximations. Here we address the chiral effective Lagrangian model proposed by Furnstahl, Serot and Tang [3], whose mean field treatment is hereafter called E-RMF model. A nuclear EFT contains all the nonrenormalizable couplings consistent with the underlying symmetries of QCD. The effective Lagrangian is expanded in powers of the fields and their derivatives, and the possible terms and their importance is systematically categorized [3, 4, 5, 6]. None of the couplings present at a given order can be arbitrarily dropped out without a symmetry argument.

The free parameters of the E-RMF Lagrangian have been optimized by fitting to the ground-state properties of a few doubly-magic nuclei [3]. The truncation of the effective Lagrangian at the first lower orders is validated by the fact that when one includes up to fourth order terms in the expansion, the E-RMF fits (parameter sets G1 and G2 determined in Ref. 31) display naturalness and the results are not dominated by the last terms retained [3, 4, 5, 6, $, 9,10,11,12]$.

Very recently, the flow of matter in heavy-ion collisions has been analyzed to determine experimentally the pressures attained at densities ranging from two to five times the saturation density of nuclear matter [14]. Nuclear collisions in man-made laboratories can nowadays compress nuclear matter to such extreme densities, which occur in Nature within neutron stars and core-collapse supernovae. Nuclear models of various sorts that are tuned to the known properties of terrestrial nuclei at normal densities or to nucleon-nucleon (NN) scattering data are commonly extrapolated for predictions of those very dense systems. The recent breakthrough achieved in the experimental determination of the equation of state (EOS) of high-density matter [14] motivates us to study the applicability of the RMF and 
E-RMF models to those conditions. The performance of the E-RMF approach for finite nuclei has already been demonstrated in a series of previous works [3, 10, 11, 12, 13].

\section{DISCUSSION}

The energy density functional of the E-RMF model is written as [3], 4]:

$$
\begin{aligned}
& \mathcal{E}(\mathbf{r})= \\
& \sum_{\alpha} \varphi_{\alpha}^{\dagger}\left\{-i \boldsymbol{\alpha} \cdot \nabla+\beta(M-\Phi)+W+\frac{1}{2} \tau_{3} R+\frac{1+\tau_{3}}{2} A-\frac{i}{2 M} \beta \boldsymbol{\alpha} \cdot \boldsymbol{\nabla}\left(f_{v} W+\frac{1}{2} f_{\rho} \tau_{3} R+\lambda A\right)\right. \\
& \left.+\frac{1}{2 M^{2}}\left(\beta_{s}+\beta_{v} \tau_{3}\right) \Delta A\right\}_{\alpha}+\left(\frac{1}{2}+\frac{\kappa_{3}}{3 !} \frac{\Phi}{M}+\frac{\kappa_{4}}{4 !} \frac{\Phi^{2}}{M^{2}}\right) \frac{m_{s}^{2}}{g_{s}^{2}} \Phi^{2}-\frac{\zeta_{0}}{4 !} \frac{1}{g_{v}^{2}} W^{4} \\
& +\frac{1}{2 g_{s}^{2}}\left(1+\alpha_{1} \frac{\Phi}{M}\right)(\nabla \Phi)^{2}-\frac{1}{2 g_{v}^{2}}\left(1+\alpha_{2} \frac{\Phi}{M}\right)(\nabla W)^{2}-\frac{1}{2}\left(1+\eta_{1} \frac{\Phi}{M}+\frac{\eta_{2}}{2} \frac{\Phi^{2}}{M^{2}}\right) \frac{m_{v}{ }^{2}}{g_{v}{ }^{2}} W^{2} \\
& -\frac{1}{2 g_{\rho}^{2}}(\nabla R)^{2}-\frac{1}{2}\left(1+\eta_{\rho} \frac{\Phi}{M}\right) \frac{m_{\rho}^{2}}{g_{\rho}^{2}} R^{2}-\frac{1}{2 e^{2}}(\nabla A)^{2}+\frac{1}{3 g_{\gamma} g_{v}} A \Delta W+\frac{1}{g_{\gamma} g_{\rho}} A \Delta R .
\end{aligned}
$$

The energy density (11) contains tensor couplings and scalar-vector and vector-vector meson interactions in addition to the standard scalar self-interactions $\kappa_{3}$ and $\kappa_{4}$. One interprets the E-RMF formalism as a covariant formulation of DFT. That is, the mean field model approximates the exact (but unknown) energy functional of the many-nucleon system, which includes all higher-order correlations, by expanding it in powers of the meson fields. The latter play the role of auxiliary Kohn-Sham potentials. The unresolved dynamics beyond mean field is encoded in the fitted coupling constants of the effective theory and the introduction of new interaction terms aims at an improved representation of the nuclear energy functional. Further insight into the concepts of the E-RMF model can be gained from Refs. [3, 4, 5, 6].

For uniform nuclear matter all of the terms with gradients in the energy density $\mathcal{E}$ vanish and the nucleon density is given by $\rho=\frac{\gamma}{6 \pi^{2}} k_{F}^{3}(\gamma=4$ for symmetric nuclear matter, $\gamma=2$ for neutron matter, and $k_{F}$ is the Fermi momentum). The pressure $P$ follows from the

derivative of the energy density with respect to the nucleon density: $P=\rho^{2} \frac{\partial}{\partial \rho}(\mathcal{E} / \rho)$.

The recent experimental observations [14, 15] rule out any strongly repulsive nuclear EOS. The constraints on the EOS of symmetric nuclear matter at zero temperature, derived 


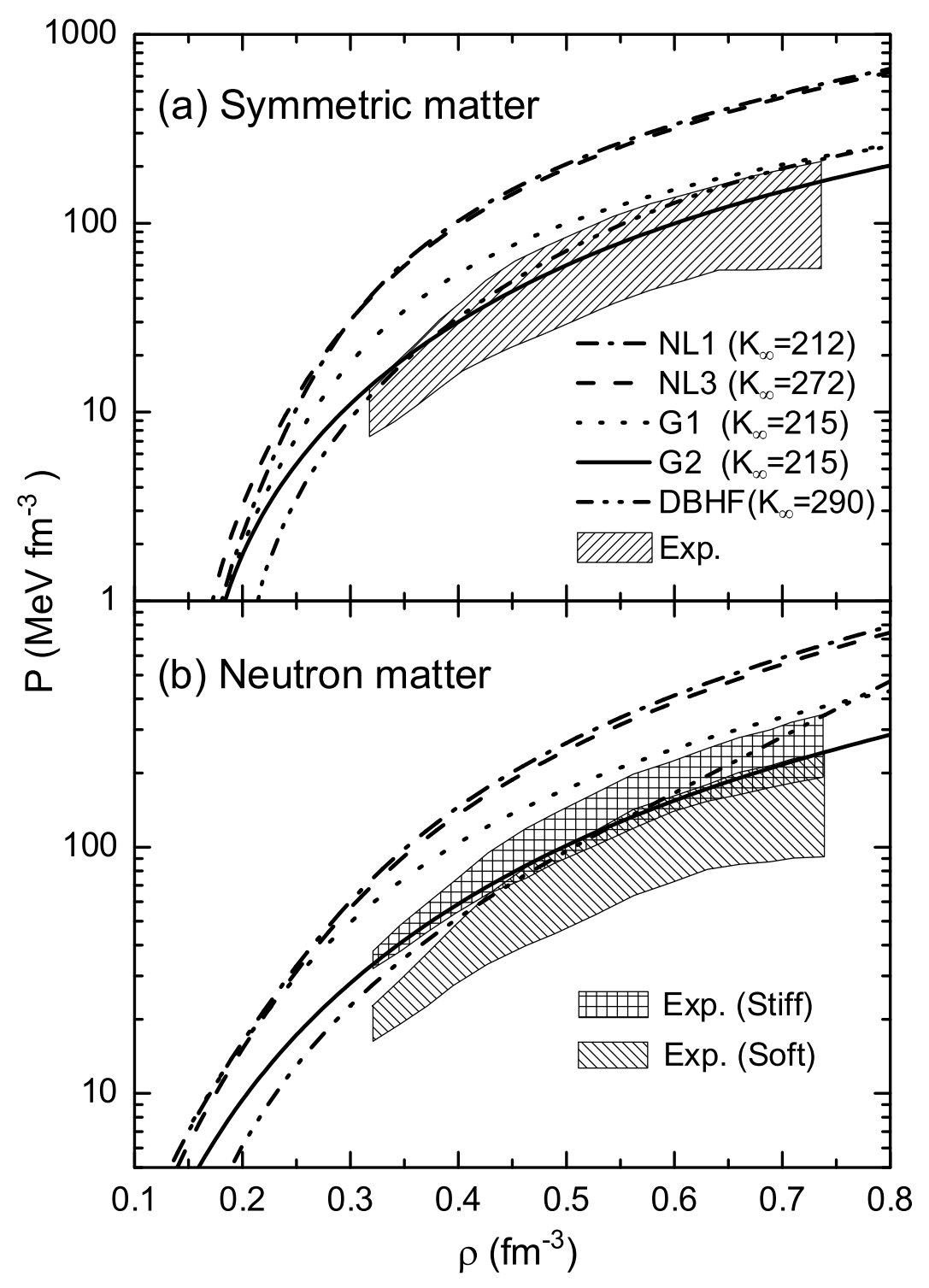

FIG. 1: (a) Zero temperature EOS for symmetric nuclear matter. The shaded area represents the region consistent with the experimental data [14]. The nuclear matter incompressibility of each interaction is expressed in $\mathrm{MeV}$. (b) Zero temperature EOS for neutron matter. The upper and lower shaded areas depict the regions compatible with experiment after inclusion of the pressure from asymmetry terms with, respectively, strong and weak density dependences [14].

experimentally by analyzing elliptic and transverse flow observables in nuclear collisions, are shown in Fig. 1a along with the predictions from different RMF and E-RMF sets. We can see that the calculations of dense matter based on NL1 [16] and NL3 17] deviate drastically from experiment, while the E-RMF calculations with G1 (to some extent) and G2 (to a better extent) agree more with the allowed region. A similar situation prevails in the EOS 
of neutron matter (Fig. 1b). The figures show that conventional sets such as NL1 and NL3 are not well suited for describing the EOS of dense matter, principally due to the fact that without the additional self- and cross-interactions the density dependence of the mesonic mean fields is too poor [10, 18]. Notice that although the incompressibility of NL1 is $K_{\infty}=212 \mathrm{MeV}$, which is within the empirical limit, the resulting EOS is stiff at higher densities and does not follow the experimental trend with increasing density. On the other hand, the E-RMF calculations explain better the situation without any forced changes in the fitted parameters or the formalism, and the consistency of G2 with experiment is outstanding. As depicted in Fig. 1, the microscopic Dirac-Brueckner-Hartree-Fock (DBHF) theory [19], which starts from the NN interaction in free space, also suggests an EOS for dense matter much softer than in the usual RMF approach. One notices that the DBHF results, for both nuclear and neutron matter, lie within the regions of the EOS compatible with the boundaries extracted from experiment.

The success of G2 in nuclear matter is possible owing to the bulk couplings $\zeta_{0}, \eta_{1}$, and $\eta_{2}$ which confer an extra density dependence to the scalar and vector self-energies. If using the functional form (11) one attemps to get a soft EOS for dense matter with vanishing $\eta_{1}$ and $\eta_{2}$, for realistic saturation conditions, this tends to enforce large unnatural values of $\zeta_{0}$. If with $\eta_{1}=\eta_{2}=0$ one keeps $\zeta_{0}$ at acceptable values, then $\kappa_{4}$ tends to become negative. With inclusion of the additional parameters $\zeta_{0}, \eta_{1}$ and $\eta_{2}$ one can agree better with experiment, have $\kappa_{4}>0$ and a not very large $\zeta_{0}$ value, as in the case of G2. The experimental data of Fig. 1 also are explained reasonably well [14] by the calculations of Akmal et al [20] which employ the Argonne $v_{18}$ interaction, though the use of this interaction for finite nuclei is still limited. It will be worth exploring the situation for relativistic models that taking advantage of density-dependent coupling vertices are consistent with the DBHF theory [21].

In Table I we present the results for the mass and radius of a neutron star. The structure of the neutron star is determined through the Tolman-Oppenheimer-Volkov equation, which follows from hydrostatic equilibrium in strong gravitational fields and general relativity [22], combined with the EOS for $P(\rho)$. One sees that the neutron star properties computed with G2 are very much in accord with the available experimental information. The critical temperatures for the liquid-gas phase transition from our calculations of nuclear matter at finite temperature are also displayed in Table I. These values are governed by the EOS around saturation where the E-RMF sets produce similar results to NL3. Hence, one expects 
TABLE I: The radius $R$ (in $\mathrm{km}$ ) and the mass ratio $M / M_{\odot}$ of a neutron star, and the critical temperature $T_{c}$ (in $\mathrm{MeV}$ ) for the liquid-gas phase transition in nuclear matter and in asymmetric matter with asymmetry $\alpha=\left(\rho_{n}-\rho_{p}\right) /\left(\rho_{n}+\rho_{p}\right)=0.2$.

\begin{tabular}{lrrrrr}
\hline \hline & NL1 & NL3 & G1 & G2 & Exp. \\
\hline$R$ & 16.94 & 15.47 & 13.88 & 10.08 & $10-12$ \\
$M / M_{\odot}$ & 2.93 & 2.78 & 2.15 & 2.05 & $1.5-2.5$ \\
\hline$T_{c}$ (Sym.) & 13.5 & 14.3 & 14.3 & 14.2 & $13-20$ \\
$T_{c}$ (Asym.) & 12.9 & 13.7 & 13.8 & 13.7 & \\
\hline \hline
\end{tabular}

that in the finite nuclei regime G1 and G2 yield results on a par with the celebrated NL3 parametrization. That this is indeed the case has been proven in previous works and therefore we shall not attempt to present more results in this respect here. We rather refer the reader to the literature [3, 9, 10, 11, 12, 13] where it has been shown that the bulk and singleparticle properties of finite nuclei are well described by the E-RMF model with a similar quality to the standard RMF parametrizations, not only for stable isotopes but also for exotic isospin-rich nuclei far from the valley of $\beta$ stability.

In choosing between the parameter sets G1 and G2 for further calculations we prefer G2. It is worth noting that G2 presents a positive value of the $\Phi^{4}$ coupling constant $\left(\kappa_{4}\right)$, as opposed to G1 and to most of the successful RMF parametrizations, such as NL3. Though the energy spectrum strictly has no lower bound with a negative $\kappa_{4}$ [23], such negative value is necessary in the standard RMF model to get the results closer to experiment. On the other hand, to have a positive $\kappa_{4}$ it is not indispensable to make two parameter sets (one for light and one for heavy nuclei) as in Ref. [18].

\section{SUMMARY}

In conclusion, we have shown for the first time that the E-RMF parameter sets give a soft EOS both around saturation and at high densities which is consistent with the measurements of kaon production [15] and the flow of matter [14] in energetic heavy-ion collisions, and with the observed neutron star masses and radii. With the systematic inclusion of new interaction terms under the guidance of EFT techniques, and without forcing any change of 
the parameters initially determined from a few magic nuclei, the E-RMF calculations with the G2 set explain finite nuclei and nuclear matter in a unified way with a commendable level of accuracy in both the cases. The authors are not aware that it has been possible to prove this for other effective nuclear models. At present, the E-RMF approach can be considered as a salient step towards a unified theory for finite nuclei as well as for infinite nuclear matter and highlights the potential of the application of effective field theory formulations for nuclear structure studies.

\section{Acknowledgments}

Work partially supported by grants BFM2002-01868 (DGI, Spain, and FEDER) and 2001SGR-00064 (DGR, Catalonia). T. S. also thanks the Spanish Education Ministry grant SB2000-0411 for financial support.

[1] B. D. Serot and J. D. Walecka, Adv. Nucl. Phys. 16, 1 (1986).

[2] J. Boguta and A. R. Bodmer, Nucl. Phys. A292, 413 (1977).

[3] R. J. Furnstahl, B. D. Serot, and H. B. Tang, Nucl. Phys. A615, 441 (1997); ibid A640, 505 (1998).

[4] B. D. Serot and J. D. Walecka, Int. J. Mod. Phys. E6, 515 (1997).

[5] H. Müller and B. D. Serot, Nucl. Phys. A606, 508 (1996).

[6] R. J. Furnstahl and B. D. Serot, Nucl. Phys. A671, 447 (2000).

[7] N. Kaiser, S. Fritsch, and W. Weise, Nucl. Phys. A697, 255 (2002).

[8] N. Kaiser, S. Fritsch, and W. Weise, Nucl. Phys. A700, 343 (2002).

[9] M. Del Estal, M. Centelles, and X. Viñas, Nucl. Phys. A650, 443 (1999).

[10] M. Del Estal, M. Centelles, X. Viñas, and S. K. Patra, Phys. Rev. C 63, 024314 (2001).

[11] M. Del Estal, M. Centelles, X. Viñas, and S. K. Patra, Phys. Rev. C 63, 044321 (2001).

[12] M. A. Huertas, Phys. Rev. C 66, 024318 (2002).

[13] Tapas Sil, S. K. Patra, B. K. Sharma, M. Centelles, and X. Viñas, Phys. Rev. C 69, 044315 (2004).

[14] P. Danielewicz, R. Lacey, and W. G. Lynch, Science 298, 1592 (2002). 
[15] C. Sturm et al, Phys. Rev. Lett. 86, 39 (2001); C. Fuchs, A. Faessler, E. Zabrodin, and Yu-Ming Zheng, Phys. Rev. Lett. 86, 1974 (2001).

[16] P. -G. Reinhard, M. Rufa, J. Maruhn, W. Greiner, and J. Friedrich, Z. Phys. A323, 13 (1986).

[17] G. A. Lalazissis, J. König, and P. Ring, Phys. Rev. C 55, 540 (1997).

[18] Y. Sugahara and H. Toki, Nucl. Phys. A579, 557 (1994).

[19] R. Brockmann and R. Machleidt, Phys. Rev. C 42, 1965 (1990); G. Q. Li, R. Machleidt, and R. Brockmann, Phys. Rev. C 45, 2782 (1992).

[20] A. Akmal, V. R. Pandharipande, and D. G. Ravenhall, Phys. Rev. C 58, 1804 (1998).

[21] S. Typel, T. v. Chossy, and H. H. Wolter, Phys. Rev. C 67, 034002 (2003), and references quoted therein.

[22] S. Weinberg, Gravitation and Cosmology, (Wiley, New York, 1972).

[23] G. Baym, Phys. Rev. 117, 886 (1960). 